\title{
XLIVE: INTEGRATING SOURCES WITH XQUERY An efficient XQuery evaluation prototype with TGV
}

\author{
Nicolas Travers \\ PRiSM Laboratory, University of Versailles, 45 av des Etats-Unis, Versailles, France \\ Nicolas.Travers@prism.uvsq.fr \\ Tuyêt-Trâm Dang-Ngoc \\ ETIS Laboratory, University of Cergy-Pontoise, 2 rue A. Chauvin, Pontoise, France \\ Tuyet-Tram.Dang-Ngoc@u-cergy.fr \\ Tianxiao Liu \\ ETIS Laboratory, University of Cergy-Pontoise, 2 rue A. Chauvin, Pontoise, France and XCalia SA, Paris, France \\ Tianxiao.Liu@u-cergy.fr,Tianxiao.Liu@xcalia.com
}

Keywords: XQuery evaluation, Extensible Optimization

Abstract: This paper presents an efficient evaluation of XQuery in a heterogeneous distributed system.

XQuery(W3C, 2005) is a rich and so a complex language. Its syntax allows us to express a large scale of queries over XML documents. We have extended (Chen et al., 2003) proposal to rewrite XQuery expressions in "canonical XQuery" in order to support the full XQuery specification.

The XQuery expressiveness makes difficulties to obtain an exclusive internal representation within a system. Models based on Tree Patterns have been proposed, and we have extended the tree pattern model to a model called TGV that (a) integrates the whole functionalities of XQuery (b) uses an intuitive representation that provides a global visualization of the request in a mediation context and (c) provides a support for optimization and for cost information.

Our paper is based on the XLive mediation system. XLive integrates sources in a uniform view. It is a running research vehicle designed at PRiSM Laboratory for assessing the integration system at every stage of the process starting from sources extraction to the user interface and is already used in several projects.

This paper presents XLive, an efficient XQuery processing in a heterogeneous distributed system.

\section{XLIVE ARCHITECTURE}

The XLive prototype is designed to be a light mediation system with high modularity and extension capabilities. It is a running research vehicle designed for assessing the integration system at every stage of the process starting from sources extraction to the user interface, including query parsing and modeling, optimization and evaluation, and also benchmarking.

As most mediation systems (Wiederhold, 1992), XLive is composed of three layers: Presentation, Integration and Sources.

Figure 1 shows the integration system. On the Source Layer, there are multiple heterogeneous data sources (relational/object/XML data sources, webservices, files, etc.) These sources are queryable by the XLive system via wrappers. The Wrapper is a component for accessing a specific Source for query-

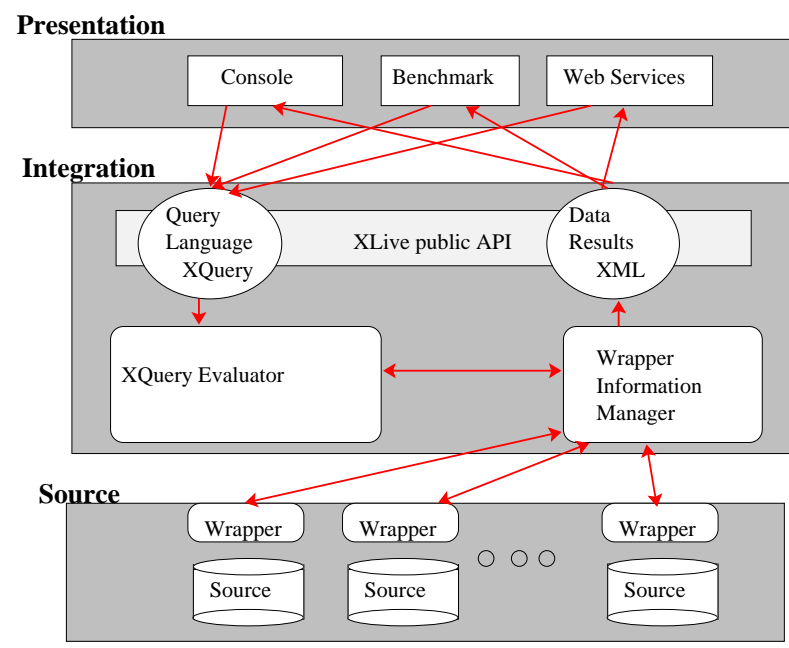

Figure 1: XLive Architecture

ing and retrieving result. As sources have specific access methods, the role of the wrapper is to translate the wrapper specificity to a common access method.

The Integration Layer is the heart of the medi- 
ation system as it process XQuery requests according to sources and send by the result in XML form. The Wrapper Information Manager is for integrating information about wrapped sources. It provides to the mediator sources metadata, capabilities, and costs statistics on source data. The XQuery Evaluator parses the XQuery query, make logical plans then physical plans by using optimization rules, choose the best execution plan, and then evaluates the execution plan by querying relevant sources and merging results The XLive system is provide a public API that process an XQuery query and evaluates it on sources, and then return the result as an XML document.

Some clients using the XLive API have been implemented on the Presentation Layer: The Console is a graphical interface for managing and querying XLive. The Web service provides services for managing/querying XLive from the Web. And finally, the Benchmark is designed to test the XLive mediator and other sources in order to make comparisons.

More details on the XLive architecture can be found on (Dang-Ngoc and Gardarin, 2003; DangNgoc et al., 2005). The TGV model used for XQuery evaluation has been described in (Travers and DangNgoc, 2006a; Travers et al., 2007). And a paper describing the TGV annotation method and extensible optimization rules has been submitted to this conference in the paper session.

\section{XQUERY EVALUATION}

XQuery is a rich and complex language. Its powerful expression capabilities provide a large range of queries over XML documents. However the richness of the language makes the evaluation of XQueries inefficient in several cases.

Figure 2 describes the evaluation process: (1) XQuery is canonized into a canonical form of XQuery (2) then the canonized XQuery is modeled in the internal structure TGV which can (3) be restructured into equivalent structures using equivalence rules. (4) Then the TGV is annotated with information for evaluation such as the data sources location, cost models information, sources functional capabilities, etc. The optimal annotated TGV is then selected and (5) the logical TGV is transformed into an execution plan using a physical algebra. We have chosen the XAlgebra (Dang-Ngoc and Gardarin, 2003), an extension of the relational algebra to XML. (6) Finally, the execution plan is evaluated and produces an XML result.

The whole process is implemented in the XLive (Dang-Ngoc et al., 2005) system and validates all usecases defined by the $\mathrm{W} 3 \mathrm{C}$ that do not imply strong

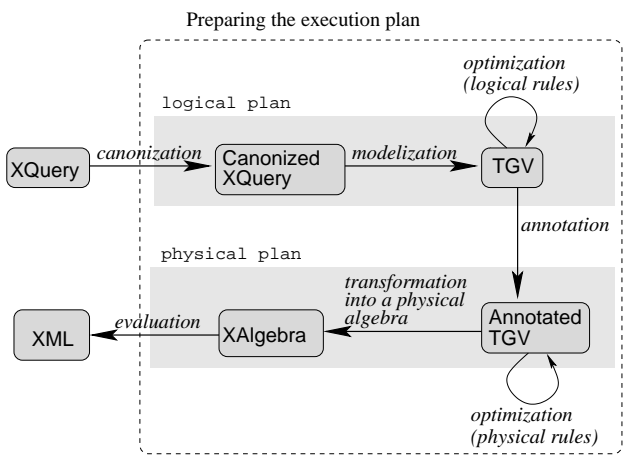

Figure 2: XQuery evaluation process

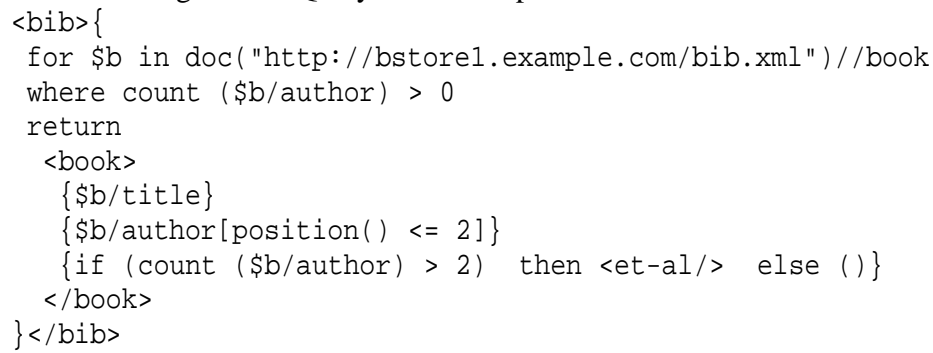

Table 1: An XQuery query example

typing consideration (our system recognize 8 of the 9 categories of XQuery).

Table 1 gives an XQuery query example supported by our XQuery processor. It is the query XMP-6 defined in the $\mathrm{W} 3 \mathrm{C}$ use-cases document. This query expresses the following request: "For each book that has at least one author, list the title and first two authors, and an empty "et-al" element if the book has additional authors."

\subsection{XQuery Canonization}

XQuery defines complex operations such as aggregation, ordering, nesting/unnesting, document construction, conditional cases (if-then-else), and XPath filter. To handle such functionalities, a canonical form using simple sequences of FOR...LET...WHERE...ORDER-BY...RETURN (FLWOR) expressions can be used (the demonstration begin in (Chen et al., 2003) and achieved in (Travers and Dang-Ngoc, 2006b)).

In order to optimize the evaluation of such queries, we propose to structure the language declaration by canonizing it using some transformation rules. These transformation rules keep the semantic of queries and make them more convenient to manipulate.

Table 2 gives the canonized XQuery form of query shown of the example presented in table 1. The following canonization rules have been used:

- Each FLWR-type queries nested in the return clause is redefined in a let clause and is assigned 


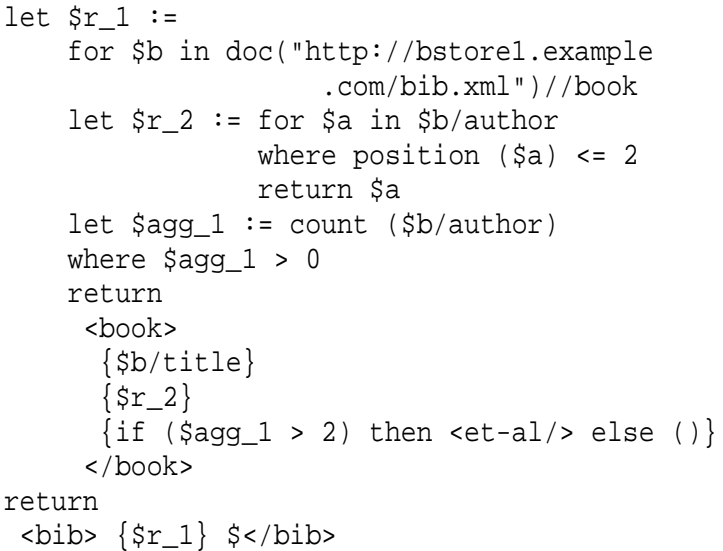

Table 2: Canonized XQuery query

to a variable. In the return clause, the nested queries are replaced by their assigned variable.

- Each aggregate function is redefined in a let clause and is assigned to a variable that replaces the aggregate function in the main expression.

- Each quantifier functions (every and some) is redefined in a let clause and is assigned to a variable that replaces the quantifier functions in the main expression.

- Nested expressions in conditional expressions If/Then/Else are declared in let clauses with new variables. The conditional expression is rewritten using those variables.

\subsection{TGV}

XQuery modeling is a difficult goal since it provides a large set of functionalities. Its canonization in a canonical form helps us defining a more simple model. Moreover, mediation purpose directs our reflection for modeling in specific Tree Patterns in which all XQuery operations are represented in a single expressive form. Each set of trees defined in canonized queries generates a particular representation.

Tree Pattern Queries (Sihem et al., 2002; Jagadish et al., 2001) are now well admitted for modeling parts of XML Queries. Works as GTP (Chen et al., 2003) use the Tree Pattern Query as a basis to model a part of the XQuery specification.

We propose the TGV (Tree Graph View) model which is implemented in the XLive mediator to process XQuery. The TGV model (Travers and DangNgoc, 2006a; Travers et al., 2007) extends the Tree Pattern representation in order to make it intuitive and full non-typed XQuery support. XQuery queries are modeled in TGV, then optimized, translated to an execution plan and evaluated.
The TGV model allows us to cover 8 of 9 use cases of the W3C (unless strong typing). In fact, specific XQuery characteristics have been integrated into the TGV model by canonization or specific elements.

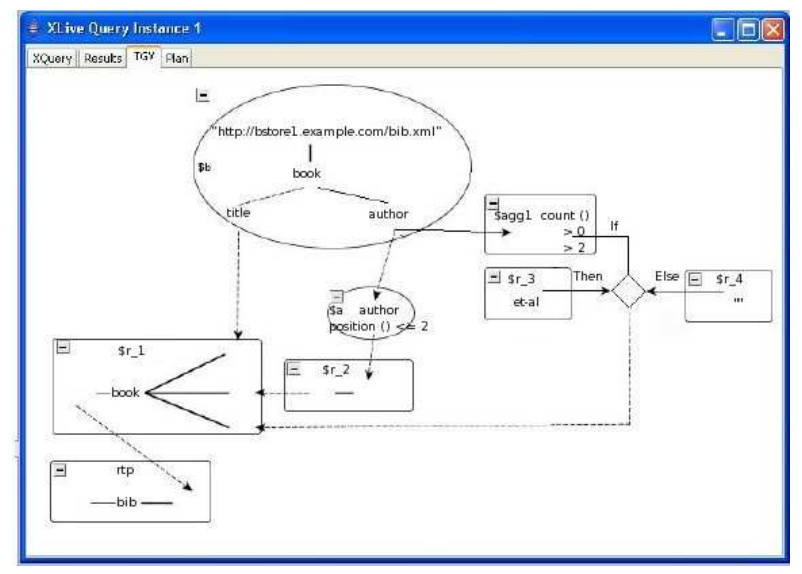

Figure 3: TGV

The TGV proposed in figure 3 illustrates the canonized query (table 2). We can identify tree patterns for source document, return document and intermediate structures. On tree patterns are represented by labels, constraints and ascendant-descendant links (simple line for descendant axis, double link otherwise), and a variable is bound. Between trees, hyperlinks represent aggregation, join, projection or condition. More description on TGV are given in (Travers and Dang-Ngoc, 2006a; Travers, 2006).

\section{THE XLIVE SYSTEM}

The whole XLive architecture with TGV support and full untyped-XQuery implementation has been released as an Open-Source software ${ }^{1}$.

In the figure 4, we show a XLive screenshot where we see (1) data sources management (2) global schema management (3) an XQuery query (4) the associated logical TGV (5) the associated XAlgebra execution plan and (6) the XML result document.

A benchmark (Dragan and Gardarin, 2005) has been created in the context of distributed semistructured model on heterogeneous sources. It has been specified and applied to XLive. It performs comparisons between queries evaluated on single sources and queries evaluated through the mediator. Mediator performance is shown in Figure 5 for a set of representative queries (Selection, projection, join, reconstruction). We use two kinds of XML data in our

\footnotetext{
${ }^{1}$ The XLive system is downloadable at http://www.prism.uvsq.fr/ ntravers/xlive/
} 


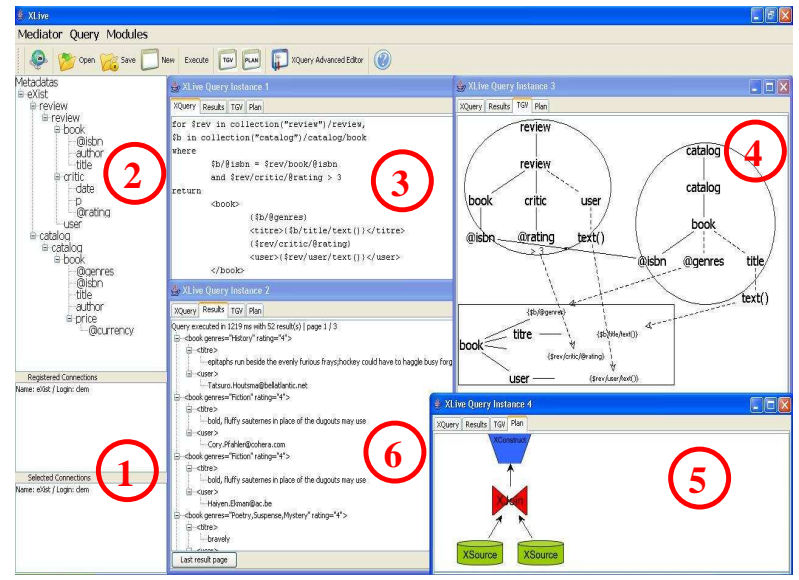

Figure 4: XLive execution

scenarios, data oriented and structure oriented. XLive stores those data in different systems: native XML repositories (XHive, Xyleme), relational systems (Oracle, MySQL) and web sources (Google, Amazon). There is an overhead due to the mediation process compared to single source evaluation. The execution is 1.4 to 2 times slower.

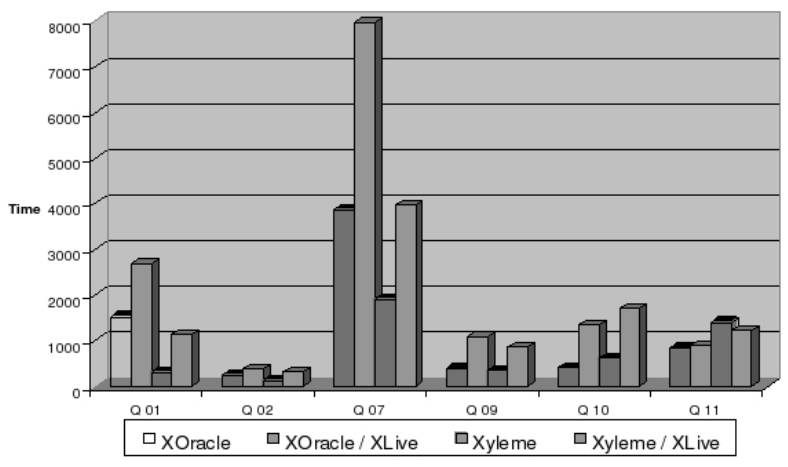

Figure 5: Benchmark

\section{ACKNOWLEDGEMENTS}

The XLive is supported by the ACI Semweb project. The cost model is supported by the ANR PADAWAN project and XCalia S.A.

\section{REFERENCES}

Chen, Z., Jagadish, H., Laksmanan, L. V., and Paparizos, S. (2003). From Tree Patterns to Generalized Tree Patterns: On efficient Evaluation of XQuery. In $V L D B$, pages 237-248, Germany.

Dang-Ngoc, T.-T. and Gardarin, G. (2003). Federating Heterogeneous Data Sources with XML. In Proc. of IASTED IKS Conf.

Dang-Ngoc, T.-T., Jamard, C., and Travers, N. (2005). XLive: An XML Light Integration Virtual Engine. In Proc. of BDA.

Dragan, F. and Gardarin, G. (2005). Benchmarking an XML Mediator. In ICEIS, Miami USA.

Jagadish, H., Lakshmanan, L. V., Srivastava, D., and Thompson, K. (2001). TAX: A Tree Algebra for XML. In DBPL, pages 149-164.

Sihem, A.-Y., SungRan, C., Laks, L. V. S., and Divesh, S. (2002). Tree Pattern Query Minimization. $V L D B$ Journal, 11(4)::315-331.

Travers, N. (2006). Extensible Optimization in an XML Mediator. $\mathrm{PhD}$ thesis, University of Versailles.

Travers, N. and Dang-Ngoc, T.-T. (2006a). Tree graph view (tgv). Technical report, PRiSM Laboratory.

Travers, N. and Dang-Ngoc, T.-T. (2006b). Xquery canonization. Technical report, PRiSM Laboratory.

Travers, N., Dang-Ngoc, T.-T., and Liu, T. (2007). Tgv: an efficient model for xquery evaluation within an interoperable system. International Journal of Interoperability in Business Information Systems (IBIS), 2. ISSN: 1862-6378.

W3C (2005). An XML Query Language (XQuery 1.0).

Wiederhold, G. (1992). Mediators in the Architecture of Future Information Systems. Computer, 25(3):38-49. 